\title{
De cadena de valor a sistemas adaptativos complejos en las industrias creativas y culturales
}

From Value Chain to Complex Adaptive Systems in Creative and Cultural Industries

Gerardo D. Neugovsen Universidad ESEADE (Buenos Aires) gerardon@gmail.com

Resumen: En este trabajo se analizan las principales características de las Industrias Creativas y Culturales como sistemas adaptativos complejos, cuyo comportamiento puede ser explicado siguiendo la Teoría de Redes, en contraste con el concepto usualmente utilizado de cadena productiva o de valor. El foco del trabajo se centra en la pregunta ¿cuál es la relación dinámica entre las industrias creativas y culturales y el resto de la economía? Se parte de comprender que las industrias creativas y culturales están constituidas por un complejo entramado de individuos y organizaciones caracterizados por una vinculación directa o indirecta con la actividad cultural y creativa, pero que a su vez estos mismos actores se encuentran vinculados de manera dinámica con otros sectores que sin ser necesariamente creativos o culturales, resultan esenciales para su efectivo funcionamiento, 
incluso cuando muchos de estos actores no son incluidos habitualmente en los análisis sectoriales. Se crean así múltiples redes de interacción en varios niveles las cuales serán analizadas en este texto. Como resultado de este análisis se hace posible mapear y comprender las relaciones dinámicas que se establecen entre los múltiples y diversos actores (stakeholders) intervinientes.

Palabras clave: industrias creativas y culturales, emprendedurismo, teoría de la complejidad, teoría de redes, sistemas adaptativos complejos, microeconomía.

Abstract: The main characteristics of the Creative and Cultural Industries are analysed as a complex adaptive system. Its behaviour can be explained following the Network Theory, in contrast to the commonly used concept of productive chain or value chain. The focus of the paper is on the question: what is the dynamic relationship between the creative and cultural industries and the rest of the economy? It is understood that the creative and cultural industries are made up of a complex network of individuals and organizations, characterized by a direct or indirect connection with cultural and creative activity, but in turn these same actors are dynamically linked with others industries, which are not necessarily creative or cultural. But those interactions are essential for their effective operation, even when many of those actors are not usually included when the creative and cultural sector is analysed. Multiple interactions are created in several levels forming a complex network, which will be analysed in this text. As a result of this analysis, it is possible to map and further understand the dynamic relationships that are established between the multiple and various intervening stakeholders. 
De cadena de valor a sistemas adaptativos complejos en las industrias creativas y culturales

Keywords: creative and cultural industries, entrepreneurship, complexity theory, network theory, complex adaptive systems, microeconomics.

Fecha de recepción: 5/4/2021

Fecha de aceptación: 9/8/2021

Neugovsen, Gerardo D. (2021). «De cadena de valor a sistemas adaptativos complejos en las industrias creativas y culturales». Monograma. Revista Iberoamericana de Cultura y Pensamiento, n. ${ }^{\circ}$ 9, pp. 81-117. doi: 10.36008/monograma.2021.09.2039. http: revistamonograma.com. ISSN: 2603-5839. 



\section{Introducción}

Las industrias creativas y culturales (ICC) constituyen un campo productivo emergente y de reciente conceptualización en el mundo académico y profesional que presenta desafíos conceptuales relevantes a las teorías administrativas existentes (Buitrago, 2013). En este trabajo se analizarán aspectos relevantes del comportamiento sectorial a nivel de microeconomía, focalizando en aquellas actividades de carácter efímero, donde el consumo ocurre cuando la actividad es ejecutada, tales como festivales, espectáculos escénicos (teatro, danza, conciertos musicales, performances, otros) y cuya realización impactará antes, durante y después de la realización de la actividad, en industrias conexas, estimulando la creación de valor en las mismas. Se busca comprender cuáles son y qué forma adquieren los vínculos entre los actores de las ICC y su relación con otros agentes económicos.

\section{Las industrias creativas y culturales: un mapa ampliado}

«La creatividad no es ninguna novedad y lo mismo cabe decir de la economía; lo que es nuevo es la naturaleza y el alcance de la relación entre una y otra y de qué forma se combinan para generar una riqueza y un valor extraordinarios»

John Howkins

Se entiende como industrias creativas y culturales (ICC) aquellas actividades económicas que se encuentran en la intersección entre las intervenciones artísticas, de creación simbólica, culturales, tecnológicas, patrimoniales y de emprendimiento y que to- 
man a la cultura, la creatividad y el conocimiento como materia prima. Se trata de un sector económicamente significativo donde, por medio de procesos productivos, sociales, tecnológicos, emprendedores y comunicacionales se generan bienes, servicios, contenidos y experiencias transables y, en muchos casos, pasibles de protección intelectual, que pueden redundar en beneficios económicos, culturales y sociales, creando (auto) empleo a través de micro, pequeñas y medianas empresas y también a través de grandes corporaciones multinacionales (UNCTAD 2008, 2010, 2013; HKU, 2010; Buitrago, 2013). El estudio The entrepreneurial dimension of the cultural and creative industries demuestra que en Europa más del 80\% de las empresas del sector corresponden a micro, pequeñas y medianas empresas de hasta 50 personas, con una fuerte preponderancia (más del 60\%) en micro y pequeñas empresas (HKU, 2010).

Las ICC adquieren diversas formas y se manifiestan a través de múltiples canales tanto tradicionales (artesanías, danzas, teatro, arquitectura, gastronomía temática, fiestas populares, ámbito editorial, cine, medios de comunicación, TV y otros), modernos (multimedios, videojuegos, apps, realidad aumentada, medios digitales, internet y otros), así como combinaciones y fusiones (procesos de convergencia, espectáculos multimediales, festivales interdisciplinarios, turismo cultural, otros). A los efectos del presente análisis se considera que las ICC operan dentro de un sistema de redes el cual puede denominarse también como un ecosistema productivo, donde intervienen múltiples actores (stakeholders) y se generan efectos económicos, sociales, comunicacionales y culturales directos, indirectos e inducidos dentro y fuera del sector, como consecuencia de la actividad generada en torno a las ICC. Tomando a las ICC como centro neuronal de la actividad, se identifican también industrias conexas que sin ser necesariamente creativas o culturales proveen insumos, equipamientos, servicios y recursos necesarios para su funcionamiento 
(hotelería, gastronomía, equipos de iluminación y audio, telas, pinturas y tintes, diversas materias prima, herramientas, servicios legales, contables, etc.), o bien se nutren de los bienes, servicios y experiencias producidos por las ICC, como es el caso de empresas productoras de eventos, el Estado, el sistema educativo y otros. Junto a esto se identifica un sector transversal que vincula a múltiples actores públicos y privados en la forma de entidades de regulación jurídica, legal y tributaria, así como también actividades comunicacionales transversales (agendas digitales de actividades, marketing digital, espacios de coworking, patrocinantes, formación y capacitación y otros), organismos de salvaguarda histórica y patrimonial y otros que vinculan varios aspectos de la actividad o que influyen en sus desempeños. Por último, el ecosistema se completa con la participación de los públicos, audiencias, clientes o similares que habrán de consumir y dotar de significado, valor y sentido a las propuestas del sector. Sin el «consumo», juicio y valoración del público, las propuestas de las ICC carecen de valor de cambio (Neugovsen, 2017).

\section{El ser y no ser de la economía en la cultura}

A pesar de ser un sector donde el principal motivo para emprender no se vincula directamente con la optimización del beneficio económico, este campo genera, sin embargo, significativos flujos económicos de manera directa, indirecta e inducida en el contexto del ecosistema antes mencionado. Se impacta así en el producto interno bruto y en la economía local de una gran mayoría de países y ciudades, superando en muchos casos los aportes de industrias tradicionales tales como las telecomunicaciones, alimenticia y otras, convirtiéndose también en un yacimiento relevante de (auto)empleo. Como consecuencia de su accionar, no solo los propios emprendimientos se benefician, sino que múltiples industrias conexas se ven dinamizadas y be- 
neficiadas generando así flujos de caja significativos dentro y fuera del sector (Stam et al., 2008; Howkins, 2001; Unesco, 2013; Kloudova et al., 2014; Pareja, 2009; Pareja et al., 2007; KEA, 2009; UNCTAD, 2008; EU Council Conclusions, 2007).

Por tratarse de un sector emergente y sumamente amplio, no existe acuerdo aún acerca de cuáles son sus fronteras, caracterizándose por la capacidad de convertir elementos simbólicos intangibles vinculados con lo estético, lo simbólico, lo emocional, lo intelectual, lo identitario y/o lo intelectual en bienes, servicios, contenidos y experiencias con valor de cambio. Respecto de quienes impulsan esta actividad, los emprendedores de las ICC, se observa que la clásica segmentación entre emprendedor por necesidad y por oportunidad (Amorós y Poblete, 2013) es trascendida, correspondiéndose más con el clásico personaje descrito por Schumpeter como:

...el fundador de una nueva empresa, un innovador que rompe con la forma tradicional de hacer las cosas, con las rutinas establecidas. Debe ser una persona con dotes de liderazgo, y con un talento especial para identificar el mejor modo de actuar. El emprendedor tiene la habilidad de ver las cosas como nadie más las ve. Los emprendedores no son managers (al estilo de Marshall) ni inversores, son un tipo especial de personas. (Schumpeter, 1934, pp. 77-78)

Relevante para este estudio es considerar lo que planteaba Schumpeter en cuanto a que este emprendedor no toma como motivación el esencialismo e individualismo del bomo economicus, guiado por la búsqueda del equilibrio entre el beneficio marginal y el coste marginal. Por el contrario, está motivado por las emociones (Goss, 2005) las cuales son, coincidentemente, uno de los principales combustibles de las ICC. Schumpeter presenta al emprendedor como un individuo que 
enfrentará hostilidades en su proceso de introducir innovaciones, dado que propondrá desviaciones de los comportamientos socialmente aceptados. Y es aquí donde encuentra una de sus principales motivaciones, en el deleite del desafío dada su condición de creativo inconformista, dinamizando (y desafiando) las fuerzas de mercado. Gracias a sus intervenciones, plantea Schumpeter, se logra romper con los flujos circulares de los procesos económicos al introducir lo nuevo, lo inesperado, sacando de su inercia a las empresas «establecidas». Se supone entonces que una de las principales motivaciones de los emprendedores de las ICC es el placer de crear algo inexistente y de desafiar en cierta medida los órdenes imperantes, dejando en evidencia cuán relevante es el contexto social en el cual se desenvuelve. En general, el dinero se convierte así en un indicador de éxito, pero no es un objetivo primario en sí mismo. Tal como indica el doctor e investigador holandés Giep Hagoort (HKU, 2010):

El principal motivador para emprender en el campo de las industrias creativas y culturales se vincula con la pasión. Todo lo demás, incluso lo económico, pasa a un segundo plano.

Lo anterior aplica también al artista o creativo, quien puede operar de manera casi simbiótica con el emprendedor de las ICC, pudiendo en muchos casos el mismo artista ser su propio emprendedor. Tal como plantea Diego Ordóñez:

La remuneración de un artista va más allá de una compensación económica. Para un artista, la proyección de su obra como realización estética, el reconocimiento de su comunidad de colegas y el reconocimiento del público, forman parte de su compensación. Por esto cada bien creativo tendrá un componente de «el arte por el arte», con implicaciones que pueden afectar la relación del artista con el comercio. Por un lado, es difícil enmarcar una obra en los cronogramas absolutos que 
requiere la planeación de proyectos cuya rentabilidad depende del tiempo. La conclusión de una obra en un intervalo de tiempo definido puede ser completamente incierta: la visión de una obra cambia, se detiene, se replantea. «La obra se hace en el camino», y no es producto de un prediseño o de una preconcepción que permita presupuestar cuánto durará su ejecución. Por otro lado, es difícil definir previamente las características de una obra. Es más, el artista pretenderá mantener independencia creativa en su trabajo y, muchas veces, sus contrapartes comerciales tendrán que confiar en la realización de un producto que, en el momento de ser comisionado, probablemente, ni siquiera el creador habrá visualizado en su totalidad. En conjunto, los altos niveles de incertidumbre y la relación del artista con su producto generan un impacto en la organización funcional de las industrias creativas y culturales y en la relación entre individuos y organizaciones. (Ordóñez, 2006)

Se trata entonces de un sector que presenta importantes diferencias conceptuales y operativas con relación a los sectores productivos tradicionales. Junto a esto, en muchos casos el «producto final» es un proceso en marcha cuyo resultado se conocerá en el momento en que el creativo o artista decida acabarlo y satisfaga sus propias exigencias. Además, le diferencia también que para el emprendedor de las ICC la generación de recursos financieros son una consecuencia del reconocimiento del «acto creativo» inicial y de la consecuente puesta en «valor de mercado» de la propuesta cultural. En este contexto «tener valor de mercado» significa que ese «acto creativo» original, habrá de adquirir valor de cambio para convertirse en un bien cultural a partir de satisfacer necesidades, deseos e intereses específicos que se vinculan con los planos emocionales, estéticos, intelectuales, identitarios, hedónicos, sociales, epistémicos (relacionado también con lo novedoso e innovador) de determinados grupos sociales, desde una perspectiva holística (Neugovsen, 2017). 
A cambio de su propuesta de valor, el creativo - mediado generalmente por la figura del emprendedor de las ICC o del gestor cultural — recibe reconocimiento, fidelización, construye capital social y económico. El emprendedor de las ICC y el gestor cultural son responsables por identificar y hacer que el acto creativo (la propuesta de valor original) circule por la sociedad para obtener valor de cambio. La puesta en valor del acto creativo ocurre en el contacto con los diversos agentes y públicos y solo cuando estos ponderan y valoran la propuesta de manera acumulativa, en la medida que logre satisfacer necesidades, deseos o intereses latentes o manifiestos. La tarea se convierte en ardua cuando se trata de propuestas de valor totalmente innovadoras o emergentes, para las cuales no existen aún públicos identificados, o cuando dichas propuestas son disruptivas.

Relevante en este análisis es el peso que posee el contexto social para la motivación y las decisiones a tomar. Schumpeter afirma que

...el liderazgo emprendedor no consiste simplemente en encontrar o crear la nueva cosa sino en impresionar lo suficiente al grupo social con ello para arrastrarlo a su estela. (Schumpeter, 1934, p. 88)

Sumado a esto, es necesario también considerar el factor tiempo, dado que muchas de las propuestas de valor de las ICC son de carácter efímero, y en donde el proceso de adjudicación de valor puede ocurrir únicamente mientras dicha propuesta es realizada y «consumida», tal como ocurre con las artes escénicas.

\section{El impacto microeconómico de la actividad cultural}

Usualmente, el análisis económico de la actividad de este sector se restringe a las evaluaciones de impacto que supuestamente 
permiten estimar los beneficios netos de una actividad determinada. Estos impactos son analizados presuponiendo relaciones causales que permitan estimar beneficios netos dentro de periodos y territorios definidos. Los objetos de estudio pueden variar desde equipos estables como museos y monumentos hasta espectáculos callejeros y afines. El foco se encuentra generalmente puesto en el valor de consumo de los «usuarios» (espectadores, público, visitantes, etc.) y se mide a partir de la supuesta disposición o voluntad de pago.

También suele incluirse el valor de consumo de los «no-visitantes», quienes pueden ser usuarios futuros influidos por temas como prestigio o beneficios a la calidad de vida. Esto agrega valores tales como opción, existencia y herencia. Junto a esto suelen medirse también los gastos a corto plazo, así como los efectos a largo plazo donde pueden estimarse incrementos de productividad así como crecimiento y desarrollo económico en la región que pueda vincularse con los activos culturales. La existencia de una tradición cultural junto a equipamientos y capital humano, hace que una ciudad sea particularmente atractiva para su visita, impactando a su vez en temas afines como los valores educativos y la creatividad (Seaman, 1987, 2003b; Bille, 1995; Howard y Crompton, 2004; Nicolas, 2010).

Junto a esto también se consideran los costos relacionados con el ambiente, la congestión, la seguridad pública, atascos de tráfico y estacionamientos, basura y otros. Por otra parte, la generalidad de los estudios económicos que se han realizado hasta el momento sobre el sector de las ICC, son de carácter macroeconómico, divulgándose cifras relacionadas con los impactos de este sector en los productos internos brutos de países y regiones. Pero en la práctica, esta información, si bien es fundamental para la consolidación como sector económicamente relevante, no ofrece información útil para los emprendedores en el día a día. 
A los efectos del presente trabajo, se busca comprender no solo cuáles son los efectos microeconómicos directos que genera la actividad cultural y creativa en un determinado territorio, sino también cuáles son los comportamientos sectoriales y de qué manera se establecen los vínculos que finalmente crean las condiciones para la generación de valor cultural, social y económico.

\section{Modelos de análisis de las ICC}

En el trabajo A new economics for creative industries and development publicado por Cunningham (2007), se presentan cuatro modelos posibles que buscan describir la compleja relación entre cultura y economía.

\subsection{Modelo 1: el «modelo de bienestar»}

Considera que las artes son económicamente exitosas en la medida que pueden extraer rentas del resto de la economía o modelo de subsidios, planteando un esquema de transferencia de recursos sustentado en argumentos de fallas de mercado. Las políticas se calibran en función de su valor de «no-mercado». En otras palabras, según este modelo, las actividades culturales tendrían un impacto negativo sobre la economía debido a que consumen más recursos de los que producen. En la medida en que existan, su valor debe estar fundamentalmente más allá del valor de mercado. Este modelo se ajusta con mayor precisión a las artes (subvencionadas) al final del espectro cultural, y la subdisciplina de la economía cultural se ha desarrollado en gran parte para abordar los problemas que surgen de estos supuestos.

\subsection{Modelo 2: el «modelo competitivo»}

Este se diferencia del anterior en que se asume que las ICC son «simplemente una industria más». Se toman como referen- 
cia los sectores de escala que se encuentran maduros, ya sea en crecimiento o en declive y que se ven afectados por aspectos de distribución debido, por ejemplo, a la creciente digitalización de procesos. Se relaciona con la industria editorial, musical, firmas de derecho de autor, medios de comunicación y otros. Las críticas de la Escuela de Frankfurt acerca de la «comoditización» de los factores culturales se orientan a estos sectores. Las políticas públicas se basan en tratamientos industriales como es el caso de multinacionales y oligopolios, buscando regular y control el exceso de control de mercado.

\subsection{Modelo 3: el «modelo de crecimiento»}

Se sustenta en una relación positiva entre las ICC y el mercado generando crecimiento. Las ICC son vistas como agentes dinámicos impulsores del crecimiento debido a que crean valor en el contexto de una economía globalizada. Se comprende que en la medida que las economías evolucionan, existen mayores posibilidades de dedicar ingresos a su consumo. En esta visión, las políticas públicas deberían adjudicar un valor especial, dado que no solo genera desarrollo inherente al sector, sino que su actividad impacta de manera positiva en el crecimiento de sectores conexos. La intervención pública debería restringirse - como en otros sectores con potencial de crecimiento- a la inversión en el crecimiento económico y en el desarrollo de la capacidad productiva que pueda dar respuesta al consumo creciente. El diseño, en su acepción más amplia, se convierte así en un factor de entrada en la economía permitiendo generar contenidos, aplicaciones (juegos, apps), medios móviles y otros. Sus efectos se ramifican hacia la educación y los nuevos paradigmas de aprendizaje, los múltiples usos industriales y recreativos de la realidad virtual en múltiples industrias no necesariamente conexas (industria aeroespacial, medicina). Diversos estudios evidencian 
la relación directa positiva entre «la intensidad de diseño en las empresas y su desempeño en el mercado de valores» (Design Taskforce 2003, Design Council 2004). En términos de creación de empleo se observa la misma correlación positiva. Haciéndose más evidente estos efectos en el crecimiento exponencial del uso de los medios móviles y de internet en la creación y usos innovadores de estos.

\subsection{Modelo 4: el «modelo de innovación» o de «economía creativa»}

De acuerdo a esta visión, la caracterización de las ICC como una industria per se, no sería totalmente adecuada, considerando al sector como un componente de los sistemas de innovación de las economías. Esta visión trasciende el análisis clásico que busca verificar la contribución relativa al valor económico (tal como lo hacen los modelos anteriores). Se parte de que el sector realiza contribuciones relevantes a la coordinación de ideas y tecnologías que a su vez dinamizan los procesos económicos y los cambios culturales. Se trataría — más que una industria en sí misma - de un sistema complejo que facilita la evolución económica derivándose de aquí su «valor económico», es decir, «un sistema que fabrica atención, complejidad, identidad y adaptación a través del recurso primario de la creatividad» (Cunningham, 2007).

De acuerdo con el modelo 4, las ICCs no impulsan el crecimiento económico de manera directa, tal como lo puede hacer un auge en el sector de los recursos primarios o en el mercado de la vivienda. En este sector ocurre de manera indirecta, a través de facilitar procesos de cambio en el orden económico. Por ejemplo, podría ser posible definir las ICCs, no como un sector industrial, sino como un espacio de actividad económica en el que los actores, los mercados y las organizaciones están predominantemente conformados por las redes sociales y, por 
lo tanto, son el tipo de «patio de maniobras» (para usar una metáfora del transporte) entre la actividad social y comercial (Potts et al., 2007). (Cunningham, 2007)

El factor diferenciador en relación con la conocida gestión cultural que basa su actividad en el patrimonio tangible o intangible existente reside finalmente en la capacidad de las ICC de desarrollar y adoptar nuevos conocimientos, orientándose hacia la experimentación y la búsqueda de lo novedoso, más que en la conservación. Evidencias de esto pueden hallarse en la creación de nuevas profesiones inexistentes hasta hace pocos años y la aparición de nuevas industrias como consecuencia de la actividad de las ICC que actúan facilitando cambios de forma y de contenido.

Como ejemplo de los modelos 3 y 4 que analizan la economía cultural, Potts et al. (2007) han propuesto recientemente una nueva definición para las industrias creativas y culturales como el espacio económico de los «mercados de redes sociales». Son el conjunto de actividades e industrias que facilitan la elección cuando se trata de la novedad en un contexto social. Estas no son solo las industrias de la información y la comunicación, ya que solo aborda la tecnología, pero más fundamentalmente son las industrias que construyen redes de personas para facilitar la acción social y la estructura, a través de las cuales pueden fluir nuevas ideas. La idea del mercado de redes sociales gana terreno al plantear que estas redes tienen propiedades características asociadas con la complejidad y la teoría evolutiva. La implicación es que esto nos ayuda a explicar por qué muchos fenómenos económicos culturales que observamos son propiedades de los modelo 3 y 4, no del modelo 1. (Cunningham, 2007)

En el mercado cultural las redes sociales de relaciones cumplen un papel tan relevante como el que ejercen las variaciones de precios en los mercados tradicionales. Interesante es observar 
que los actos de elección de consumo en las ICC están regidos menos por factores convencionales vinculados con la demanda, pero sobre todo, y de manera incremental, por las influencias mutuas que se ejercen los diversos actores a través de los medios digitales, móviles e interactivos. Las elecciones individuales se ven más influidas por otras elecciones de pares en un contexto de red social, que por la novedad artística o creativa. A modo de efecto contagio, los pagos realizados por un agente de dicha red opera como acción representativa de los otros miembros de la red. De acuerdo con Castronova (2006), las redes operan como mercados en sí mismos, que a su vez conciben nuevos mercados emergentes producto de las dinámicas no comerciales y relacionales entre los miembros. Los espacios de las plataformas de redes sociales (Facebook, YouTube, Whatsapp, Twitter y otros) emergen entonces como ámbitos de flujo donde no se adquiere la propiedad, sino que el espacio social es el lugar donde se generan las interacciones que darán lugar a los procesos comerciales.

El modelo 4 analizado previamente, permite suponer que la fortaleza de las ICC no reside tanto en su capital cultural o su valor no-comercial, sino que todas las etapas relacionadas con la producción, distribución y consumo transcurren a través de redes sociales complejas y en donde los niveles de confianza en el boca en boca, en las tendencias y en la reputación son mucho mayores que en otras industrias. La puesta en valor de las propuestas de las ICC ocurre, como se señalaba previamente, debido a las múltiples retroalimentaciones de información que sobre un determinado bien, servicio o experiencia, circulan a través de las redes sociales, mucho más que las cualidades intrínsecas o los precios. Analizar la economía cultural o creativa implica entonces reconocer que no se trata de la reutilización de los recursos, como plantea el modelo 1 , sino que se relaciona con redes sociales dinámicas, de carácter abierto y autopoiéticas, estableciendo así condiciones apropiadas para generar crecimiento, bienestar, cambio e innovación. 
Debido a que diversos agentes participan en menor o mayor medida de los procesos creativos - como en el caso de los públicos- los modelos existentes relacionados con las estructuras económicos (neo)clásicas y de consumo se ven alterados, modificando la percepción acerca de los modos en que ocurren los intercambios en los mercados culturales y de las ICC. Surge entonces la cuestión acerca de cómo se conforman estas redes sociales y de qué manera se articulan sus componentes.

\section{6. «Ecosistemas productivos» en el campo cultural y creativo: síy solo si}

De lo anterior, se desprende que el tratamiento analítico del sector de las ICC como sector productivo debe considerar estas particularidades, en la medida que, tal como se ha señalado, tanto desde el lugar productivo, de distribución como de consumo, los comportamientos poseen especificidades que no se condicen con las esperadas en las tradiciones del pensamiento neoclásico. Esto lleva a formular preguntas de investigación tales como: ¿pueden ser analizadas las ICC en términos de cadenas de valor tal como se analizan otros sectores productivos? De no ser así, ¿bajo qué criterios conceptuales se deberían analizar los procesos en el campo de las ICC?

A los fines del presente trabajo se tomarán como sujeto de análisis los procesos que acontecen en el contexto de actividades escénicas, festivales y afines, una de cuyas características principales es su condición de ser efímeras y donde el consumo ocurre durante la ejecución de la obra. Si nos ubicamos mentalmente en el escenario físico de una sala de espectáculos en una ciudad y asumimos que en un determinado momento se presentará un grupo de actores, habremos de considerar que los flujos económicos que fluyen en ese primer nivel se corresponden con los ingresos que habrán de percibir los artistas, su representante y el 
De cadena de valor a sistemas adaptativos complejos en las industrias creativas y culturales

productor, el cobro de los eventuales derechos autorales sobre las obras presentadas, la venta de soportes relacionados (cedés, vídeos físicos o digitales) y eventualmente el beneficio derivado por exposición de marca que puedan obtener las empresas patrocinantes (véase área azul en la figura 1).

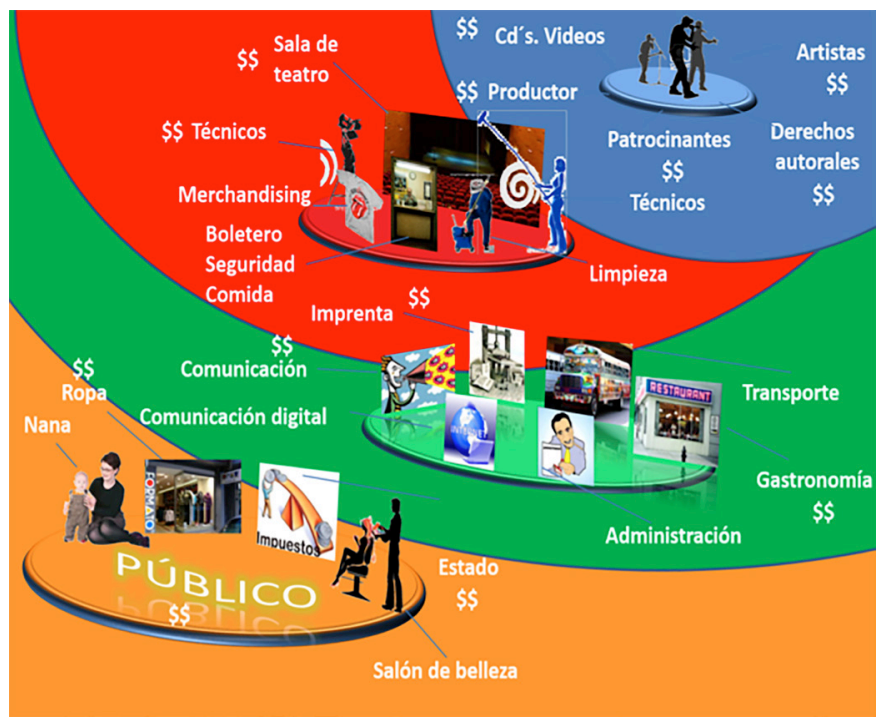

Figura 1. Ecosistema productivo industrias creativas.

En un segundo nivel de análisis, y en el arco de tiempo que transcurre la función, encontramos la actividad inherente al teatro: venta de entradas, seguridad, técnicos (sonido, iluminación, multimedios, tramoyistas, otros), vestuaristas, maquilladores, visagistas, venta de alimentos y de merchandising y otros (véase área roja en la figura 1).

En un siguiente nivel, encontramos las actividades que se vinculan con la presentación antes, durante y después de producirse la misma. Previamente tendremos la actividad de diseñadores gráficos, arquitectos, diseñadores de moda, community

Neugovsen, Gerardo D. (2021). «De cadena de valor a sistemas adaptativos complejos en las industrias creativas y culturales». Monograma. Revista Iberoamericana de Cultura y Pensamiento, n. ${ }^{\circ}$ 9, pp. 81-117. doi: 10.36008/monograma.2021.09.2039. http: revistamonograma.com. ISSN: 2603-5839. 
managers, abogados, contadores, imprentas, prensa, transporte, escenógrafos, eventualmente hotelería y otros. Durante la presentación tendremos un incremento en la actividad de transporte público y privado, estacionamientos y otros. Al concluir la función, se incrementará la actividad gastronómica en bares y restaurantes aledaños, de transporte público y privado y otros (véase área verde en la figura 1).

Finalmente y en relación directa con la actividad en el escenario y en ámbitos que se benefician indirectamente, tendremos el salón de belleza al cual se acude debido a la actividad escénica; probablemente ocurra la adquisición de una prenda de vestir para la ocasión, beneficiando a la tienda de ropa; la contratación de una persona para cuidar a los niños en la noche de la actividad; el trabajo de los contadores y administradores y, finalmente, el Estado, el cual habrá de percibir ingresos en concepto de impuestos en la mayoría de los rubros, así como cargas sociales y otros. Todas estas actividades se ven notoriamente incrementadas debido que tiene lugar la presentación escénica en las noches consideradas.

Sumado a lo anterior, debemos considerar el incremento de actividad inducida en las múltiples industrias conexas que proveen de servicios, insumos, equipamientos y otros a los diversos segmentos señalados, las cuales verán incrementada su actividad debido a que en la sala se producen espectáculos artísticos en un periodo de tiempo determinado. Encontramos aquí desde la provisión de insumos para indumentaria, escenografía, merchandising, maquillaje, gastronomía, equipamientos de iluminación, audio y audiovisuales, tintas y un largo etcétera que se encuentran en relación directa con la producción del espectáculo. Todo este incremento de actividad productiva ocurre sí y solo si debido a la presentación de la obra. Si la obra no se realiza, gran cantidad de estas actividades son «anuladas» percibiendo impactos negativos en sus economías. 
En otras palabras, la producción de un espectáculo escénico - una de cuyas características como se ha mencionado, es la de ser efímera- genera un relevante movimiento productivo a nivel microeconómico, activando múltiples industrias que no necesariamente son creativas, pero que se ven beneficiadas por esta actividad. Si a su vez potenciamos este análisis con la cantidad de salas y espacios donde se presentan actividades artísticas y culturales en una ciudad en un determinado momento y lo relacionamos con todas las actividades productivas conexas que se dinamizan a causa de las presentaciones artísticas y culturales, se obtendrán redes de interacción productiva de alta complejidad que dinamizan no solo la vida económica del territorio analizado, sino que también inciden en la calidad de vida del espacio estudiado generando impactos relevantes en los patrimonios tangibles e intangibles de ese territorio.

\section{Caso: Festival Internacional de Cine de San Sebastián}

Estudios microeconómicos realizados en torno a festivales de cine como el de San Sebastián, en el País Vasco o el Festival de Cine de Valdivia en Chile (Devesa et al., 2012) ponen en evidencia los flujos directos originados por la actividad cultural (venta de entradas, contratos por derechos, venta de merchandising del Festival y la generación de empleo permanente y temporario). También se revelan los impactos indirectos e inducidos que ambos festivales generan a nivel de industrias conexas localmente, como en la hotelería, la gastronomía, el transporte, el merchandising y la imagen a nivel global de la ciudad. Por último, se evidencian también los flujos relacionados con los dineros públicos que, por un lado, se aportan para la realización de las actividades en forma de subsidios pero que retornan en forma de impuestos, cargas sociales y otros a través de las múltiples transacciones que tributan

Neugovsen, Gerardo D. (2021). «De cadena de valor a sistemas adaptativos complejos en las industrias creativas y culturales». Monograma. Revista Iberoamericana de Cultura y Pensamiento, n. ${ }^{\circ}$ 9, pp. 81-117. doi: 10.36008/monograma.2021.09.2039. http: revistamonograma.com. ISSN: 2603-5839. 
impositivamente y abonan sus respectivas cargas sociales e impositivas. Todo lo cual resulta, en el caso de San Sebastián, en que el retorno impositivo indirecto percibido por el Estado es superior al valor del subsidio otorgado a los organizadores. Junto a esto, se genera además valor intangible de marca-país, prestigio, confianza y orgullo entre sus habitantes, mejorando notoriamente la percepción de estos centros urbanos a nivel global como destinos turísticos y de actividades de conocimiento. Más de 1000 periodistas de 500 medios de comunicación globales asisten al Festival y reportan en sus respectivos países (El País, 2013). En otras palabras, las lógicas de generación de flujos de caja de los emprendimientos de las ICC no siguen una lógica lineal de compraventa, sino que dicho emprendimiento se convierte en el nodo de un sistema de beneficios para múltiples actores relacionados de manera directa, indirecta e inducida que debe ser activado y liderado por el emprendedor de las ICC, coincidente con el modelo 4 analizado previamente.

Festival de cine de San Sebastián 2013, País Vasco PyMe

724 empleos directos y 235 indirectos

157.256 espectadores.

2.231 profesionales de la Industria de 69 países.

996 periodistas acreditados de 42 países.

Presupuesto de 7.5 millones de euros.

Subvenciones del Estado 4.2 millones

Facturación: 45,5 millones de euros

Movimiento económico de más de 72 millones de euros

Retorno impositivo: 4.662 .583 de euros

Impuesto sobre las Personas Físicas (IRPF): 801.499 euros.

Impuesto sobre Sociedades: 746.013 euros.

Impuestos sobre Productos: 1.529.793 euros.

Seguridad Social: 1.585 .278 euros

Impacto Inducido 27.319.821 euros

61 FESTIVAL DE SAN SEBASTIÁN DONOSTIA ZINEMALDIA

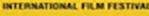

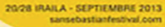

Figura 2. Datos microeconómicos Festival Cine San Sebastián. 
8. Sistemas adaptativos complejos y redes sociales: del acceso a los recursos y el análisis de los vínculos

«Los analistas de redes parten de la simple, pero poderosa noción de que la principal tarea de los sociólogos es estudiar la estructura social... El modo más directo de estudiar una estructura social es analizar la pauta de los lazos que vinculan a sus miembros. Los analistas de redes buscan las estructuras profundas, los modelos regulares de redes que se encuentran debajo de la frecuentemente compleja superficie de los sistemas sociales... Se considera que los actores y su conducta están constreñidos por esas estructuras. Por tanto, la preocupación es el constreñimiento estructural, no los actores voluntaristas»

Barry WeLLMAN

Uno de los principales objetivos de este sistema de relaciones se orienta a facilitar el acceso a recursos tangibles e intangibles que hagan posible la producción teatral, en este caso.

El elemento ordenador es la ejecución de la actividad cultural, sea este un festival, una presentación teatral o musical o similar (sí y solo si). Para lograrlo, uno de los principales agentes dinamizadores es el emprendedor de las ICC o gestor cultural, quien habrá de definir y establecer múltiples vínculos, creando redes complejas a fin de que los recursos tangibles e intangibles necesarios para que dicha actividad pueda ser realizada, estén disponibles en tiempo y forma. Se establece así un sistema complejo con agentes diversos que irá adaptándose a medida que se sucedan las múltiples actividades, relacionados con actividades de producción, distribución, de infraestructura, de comunicación y de difusión y consumo A su vez estos agentes establecen relaciones en diversos niveles con otros proveedores y agentes de industrias conexas que resultan impactados en diversos grados por la actividad nuclear a ejecutarse en una fecha, horario y lugar predeterminado.

Neugovsen, Gerardo D. (2021). «De cadena de valor a sistemas adaptativos complejos en las industrias creativas y culturales». Monograma. Revista Iberoamericana de Cultura y Pensamiento, n. ${ }^{\circ}$ 9, pp. 81-117. doi: 10.36008/monograma.2021.09.2039. http: revistamonograma.com. ISSN: $2603-5839$. 
El emprendedor de las ICC o gestor es uno de los agentes centrales en este sistema complejo y es quien debe asegurar que cada insumo, actor, equipamiento o espacio se encuentren disponibles en tiempos y espacios también previamente determinados, así como los recursos económicos relacionados. Pero su relevancia es compartida con otros agentes que inciden en el adecuado desarrollo de las actividades (véase figura 1).

Tratándose de un proceso dinámico, esta red de redes irá mutando conforme se avanza en el tiempo y en donde las relaciones que se establecen directa, indirecta e inducidamente incidirán en la calidad de los procesos, más allá de las metas concretas a alcanzar en los diversos momentos.

Así, los acuerdos realizados con los vestuaristas para que los vestuarios se encuentren disponibles a la hora del ensayo general, previo a la noche del estreno, implica a su vez que estos deben acordar con sus múltiples proveedores tiempos, cantidades y lugares de entrega en una serie concatenada de distribución y entrega de insumos. Todo lo cual a su vez depende de los diseñadores de indumentaria, quienes deben haber establecido acuerdos con el director y el productor para definir diseños y presupuestos, a su vez vinculados con contadores, generándose así múltiples relaciones que a su vez se cruzarán con otros actores relevantes como los escenógrafos, iluminadores, sonidistas y otros. De manera simultánea, los agentes responsables de la comunicación iniciarán sus estrategias comunicacionales por vías tradicionales y digitales, generando expectativa e interés en los públicos destinatarios, quienes a su vez apartarán tiempo y dinero para asistir a la función y funcionarán a su vez como comunicadores y difusores de la actividad, incidiendo en las decisiones de otras personas, tal como se ha visto previamente.

Una pregunta relevante y válida se relaciona con la identificación de cuáles son los factores motivadores que logran in- 
volucrar a un conjunto relativamente numeroso de personas y organizaciones en una actividad que no tendrá continuidad en el tiempo, ya que, una vez concluida la temporada, lo más probable es que concluyan también las presentaciones de esa obra.

El análisis desde la perspectiva económica explicaría parcialmente dichas motivaciones, siempre que consideremos que uno de los móviles principales para la oferta de servicios sea la remuneración económica. Sin embargo, tal como se ha analizado anteriormente, en general el principal móvil para trabajar en este sector, sobre todo en los ámbitos más cercanos a la producción artística, es la pasión y en donde las retribuciones monetarias son vistas como el reconocimiento a la calidad y la trayectoria, antes que como un objetivo en sí mismo. Es aquí donde se hace apropiado introducir el análisis desde la perspectiva sociológica, en búsqueda de elementos que permitan comprender los comportamientos que se hacen a su vez extensivos a los «consumidores» — el público— cuyo comportamiento difícilmente pueda explicarse únicamente desde la perspectiva económica de consumo según pautas individualistas.

El análisis anterior evidencia la existencia de secuencias concatenadas de acciones y actividades pero que no parecen responder a una estructura premeditada o planificada. La intencionalidad de un artista al presentarse en un escenario seguramente no se relaciona directamente con el incremento de comensales en un restaurante aledaño luego de la función o con la venta de telas y atributos para los vestuarios, pero estos efectos ocurren debido a la realización de la actividad cultural, generándose beneficios múltiples. Como se ha visto también, el subsidio que otorga el Estado para la producción artística, retorna a sus arcas por medio de impuestos y cargas sociales. Una lectura lineal e incompleta de los ciclos de flujo de recursos económicos impide identificar los recorridos que realizan los mismos. Se hace ne- 
cesario por lo tanto implementar una metodología analítica que haga posible la comprensión e identificación de estos flujos, de la totalidad de los actores involucrados y de los beneficios que directa, indirecta e inducidamente generan las ICC.

\section{El individualismo metodológico racional versus la sociología analítica}

Entre los especialistas se identifica un debate académico relevante en relación con los modos de explicar las relaciones descritas. Por un lado, se encuentran quienes defienden el abordaje desde el individualismo metodológico racional que propone que los fenómenos sociales - estructura y cambios - se explican a partir de las propiedades que caracterizan a los individuos involucrados, tales como metas, objetivos, creencias y acciones. Este abordaje se vincula a su vez con la escuela neoclásica de la economía basada en la maximización racional de utilidades del individuo, o costo-beneficio. Sin embargo, por lo visto en el párrafo anterior, las necesidades que satisface la cultura y su actividad no pueden valuarse numéricamente, dependiendo de análisis subjetivos, tal como lo plantea la escuela austriaca de economía (Von Mises,1949; Menger, 2007). En el análisis cuantitativo estándar, no existe un esfuerzo sistemático por mostrar cómo la relación entre variables es producida por mecanismos sociales precisos (Barbera, 2006).

En contraposición, la sociología analítica busca explicar los mecanismos a través de los cuales se producen determinados hechos sociales que se relacionan con las acciones de las personas en el contexto de las relaciones que vinculan a los diversos agentes. Se introduce así abordajes tales como la teoría de la complejidad, los sistemas complejos adaptativos y la teoría de redes sociales. 
La teoría de la complejidad busca comprender cómo las organizaciones o empresas logran adaptarse a sus contextos de intervención y hacen frente a situaciones de incertidumbre. Debido a que se trata de redes dinámicas con niveles variados de interacción entre sus componentes, se plantea que son complejas. Asimismo, la agregación de los actores individuales deriva en agregaciones que no son el resultado directo de cada elemento individual. Debido a que los comportamientos, relaciones e interacciones irán mutando con el correr del tiempo, autoorganizándose, se define el principio de adaptabilidad como una respuesta a los cambios que irán ocurriendo. Algunas características que definen los sistemas complejos adaptativos son: la autoorganización, la complejidad, la interdependencia, la coevolución y el coaprendizaje, el caos, la incertidumbre y otros. Otro factor determinante que le distingue de los sistemas ordenados y lineales se vincula con las relaciones existentes entre los diversos agentes involucrados. Las restricciones propias de los sistemas ordenados no existen en estos sistemas, autoorganizándose y generando aprendizajes que les diferencia de otros sistemas similares (Diment, Yu, Garrety, 2012; Phister, 2010; Chan, 2001). La complejidad tiene como resultado la generación de interrelaciones, interacciones e interconectividad entre elementos dentro de un sistema y entre el sistema y su contexto. Por lo mismo, poseen la capacidad dinámica de adaptarse y evolucionar con el entorno cambiante.

Por otra parte, las redes sociales se centran en una «visión de la estructura social como conjunto de vínculos que unen tanto a miembros individuales como a colectivos de la sociedad» (Requena Santos, 2003). La teoría de redes sociales provee un abordaje que hace posible esbozar el análisis de estos comportamientos. De acuerdo con Barnes, la totalidad de la vida social debería ser vista «como un conjunto de algunos puntos (nodos), vinculados por líneas para formar redes totales de relaciones. La 
esfera informal de relaciones interpersonales se verían como una parte, una red parcial de una total» (Barnes J. A., 1954: 43). El abordaje desde la sociología analítica permite comprender de qué manera los patrones de vinculación asignan los recursos en un sistema social como el que se está analizando aquí. De acuerdo a los analistas estructurales, una red social también puede ser definida como «un conjunto de lazos que vinculan a los miembros del sistema social a través, y más allá, de las categorías sociales y los grupos cerrados» (Wellman, 1988). Será Simmel quien plantee que «las relaciones sociales de un tipo concreto siguen modelos derivados de las características de una situación determinada. [...] La forma y el modelo que toman las relaciones son, incluso, más importantes que su contenido» (Requena Santos, 2003).

\section{Informática y la aplicación práctica de los conceptos analizados}

Tomando en cuenta los temas analizados, y sustentando el enfoque analítico en la teoría de redes y de la complejidad, se tomó la situación analizada inicialmente (ver figura 1) y se realizó una simulación de relaciones utilizando para esto el software NodeXL que permite realizar estudios de comportamientos complejos en redes sociales. Se definieron los siguientes actores como relacionados directos, indirectos e inducidamente que intervienen en distintas etapas en la realización de un espectáculo artístico en una sala imaginaria y en el territorio de una ciudad promedio. A los fines de la presentación se utilizó el algoritmo de Haren-Koren Fast Multiscale que permite realizar una visualización con posiciones de clústers superpuestas, permitiendo esto una adecuada comprensión de las relaciones establecidas. Este algoritmo suele utilizarse para grandes cantidades de datos, como es el caso de los estudios de redes sociales digitales tales como Twitter o Facebook. 
De cadena de valor a sistemas adaptativos complejos en las industrias creativas y culturales

TABLA 1. Actores definidos para el diseño de las redes de las figuras 3 y 4 .

\begin{tabular}{|c|c|}
\hline abogado grupo & patrocinadores \\
\hline abogado sala & prensa \\
\hline agente de prensa & proveedor equipo sonido \\
\hline artistas & proveedor equipos iluminador \\
\hline asistente director & proveedor equipos sonido grupo \\
\hline autor de la obra & proveedor escenógrafo \\
\hline bares aledaños & proveedor insumos bar \\
\hline boletería & proveedor insumos limpieza \\
\hline community manager grupo & proveedor internet grupo \\
\hline community manager sala & proveedor internet sala \\
\hline contador sala & proveedor vestuarista \\
\hline director obra & proveedor visagista \\
\hline diseñador gráfico grupo & proveedores insumos bares \\
\hline diseñador gráfico sala & proveedores insumos hoteles \\
\hline distribuidor afiches & proveedores insumos restauran- \\
\hline emprendedor IC & público \\
\hline escenógrafo & redes sociales \\
\hline estacionamientos pagos & registro derechos autorales \\
\hline Estado-recaudación tributaria & representante grupo \\
\hline Estado-subsidios a la obra & responsable bar \\
\hline estudio grabación grupo & restaurantes aledaños \\
\hline hoteles & salón de belleza \\
\hline iluminador & sonidista \\
\hline imprenta grupo & taxis \\
\hline imprenta sala & tienda de ropa \\
\hline limpieza & transporte público \\
\hline mantenimiento sala & Uber \\
\hline medios de comunicación & vestuarista \\
\hline merchandising & visagista \\
\hline niñera & \\
\hline
\end{tabular}

Neugovsen, Gerardo D. (2021). «De cadena de valor a sistemas adaptativos complejos en las industrias creativas y culturales». Monograma. Revista Iberoamericana de Cultura y Pensamiento, n. ${ }^{\circ}$ 9, pp. 81-117. doi: 10.36008/monograma.2021.09.2039. http: revistamonograma.com. ISSN: 2603-5839. 
Se ha buscado cubrir un espectro amplio de actividades según la experiencia del investigador en materia de producción de espectáculos. Como resultado se ha obtenido el siguiente esquema de redes.

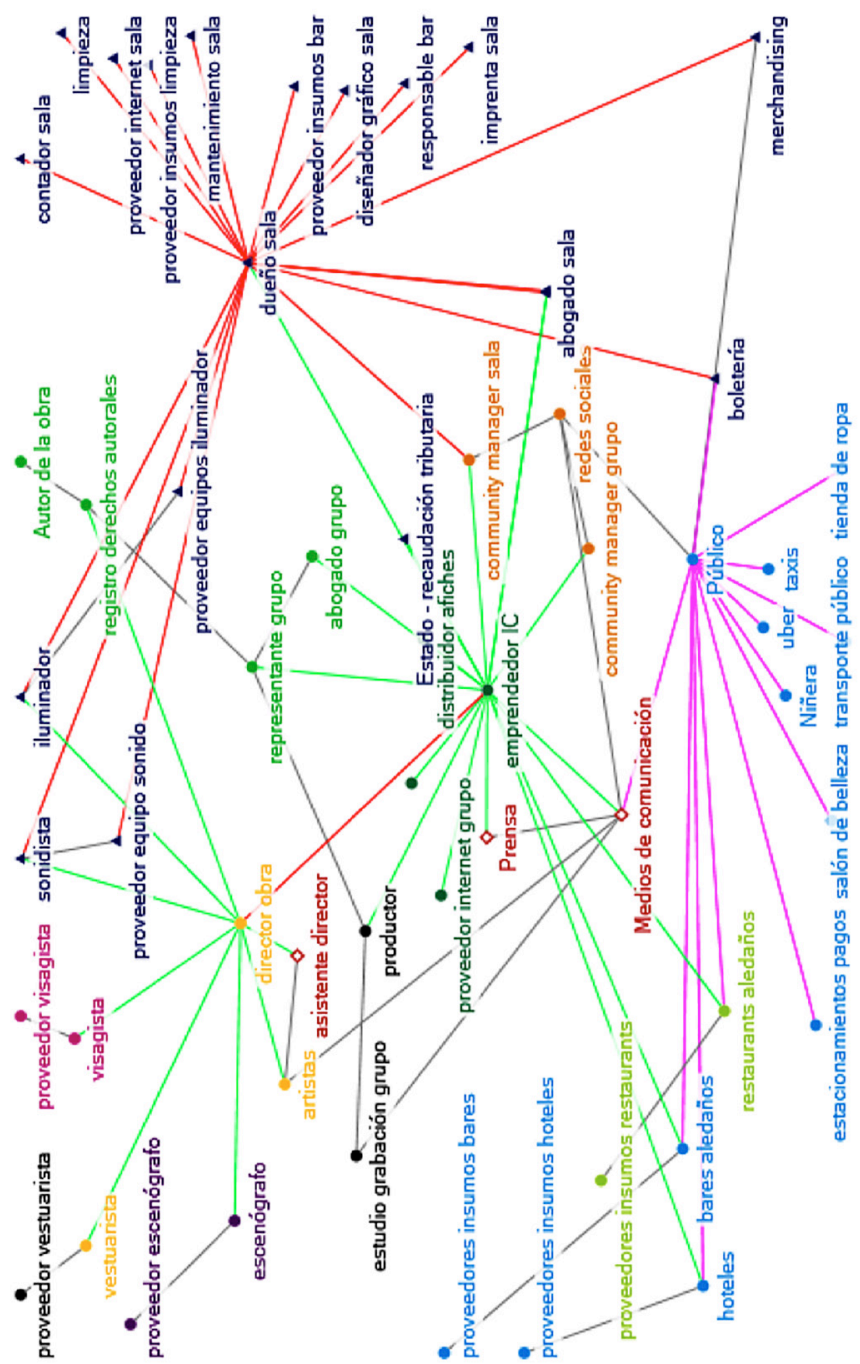

FIgURA 3. Redes sociales complejas en torno a una producción escénica.

Neugovsen, Gerardo D. (2021). «De cadena de valor a sistemas adaptativos complejos en las industrias creativas y culturales». Monograma. Revista Iberoamericana de Cultura y Pensamiento, n. ${ }^{\circ}$ 9, pp. 81-117. doi: 10.36008/monograma.2021.09.2039. http: revistamonograma.com. ISSN: 2603-5839. 
La figura 3 presenta evidencias de que existen al menos cuatro nodos relevantes determinados por la cantidad de vínculos que establecen a los fines de la realización de la producción artística a saber:

a) El emprendedor cultural.

b) El dueño de la sala de espectáculos.

c) El público.

d) El director de la obra.

En torno a estos cuatro nodos principales se despliegan los múltiples actores cuya relevancia irá modificándose a medida que el proceso de producción tiene lugar. Esta visualización permite comprender la complejidad que se esconde detrás de toda producción de espectáculos. También este mapeo permite visualizar cuáles son las relaciones que se establecen con agentes que no están directamente involucrados con los procesos culturales o creativos, pero que son necesarios para su realización.

Es posible observar que de cada actividad realizada el Estado ingresa a sus arcas tributaciones cuya recaudación se vincula directamente con la realización de la actividad artística-cultural. Lo cual plantea signos de interrogación acerca del concepto de subsidio al sector cultural, percibido como gasto de dinero público que, a juzgar por lo indicado en este mapeo, en realidad deberían ser vistos como inversiones en un sector que dinamiza la economía y genera valor económico, cultural y social.

Por último, por tratarse de una simulación basada en la experiencia, se tiene conciencia de que el mapa real de vínculos incluye a otros actores que no se han indicado aquí. Se ha buscado representar de la manera más fidedigna posible la realidad vinculada con la producción de un espectáculo. 


\section{Conclusiones}

«El mundo está compuesto por redes, no por grupos»

Barry WeLLMAN

El trabajo presentado permite extraer diversas conclusiones relevantes:

1. La posibilidad de visualizar al conjunto de actores y sus vínculos hace posibles lecturas sistémicas de la realidad productiva de las ICC, en este caso en el campo de la producción escénica en particular, permitiendo esto identificar y establecer relaciones productivas novedosas con los diversos actores involucrados. Los modelos de negocios basados en las lógicas colaborativas encuentran en este mapeo sustento y argumentos innovadores para su desarrollo.

2. La metodología utilizada permite realizar la trazabilidad microeconómica de cada segmento analizado, pudiéndose así generar información económica relevante para los emprendedores del sector, que pueden comprender el funcionamiento de su entorno de operación y tomar decisiones sobre esta información.

3. Esta información también es útil para el diseño de políticas públicas ya que al aplicar este modelo al conjunto de actividades que se realizan en una ciudad, permite obtener una visión sistémica de los procesos relacionados con cada subsector productivo de las ICC pudiendo así realizar diagnósticos y planificaciones sobre la base de la información generada. Por lo mismo, este esquema ayuda a comprender que los recursos públicos destinados al campo de la cultura deben ser vistos como inversiones y no como costos. 
4. Los modernos sistemas de big data pueden ser utilizados para generar información en tiempo real, que también permiten realizar el seguimiento de los consumos culturales.

5. En los gráficos no se indican aquí grados de relevancia de cada actor. Por el contrario, se ha mantenido una lógica equitativa para todos y cada uno de los actores.

En suma, la aplicación de la teoría de redes y de los conceptos de sistemas complejos adaptativos resulta apropiada para comprender los comportamientos microeconómicos del sector de las industrias creativas y culturales. 


\section{REFERENCIAS BIBLIOGRÁFICAS}

ANHeIER, H. e IsAR, Y. R. (2008). Cultures and globalization: the cultural economy. Londres: SAGE.

Barbera, F. (2006). «A Star is Born? The Authors, Principles and Objectives of Analytical Sociology». Papers. Revista de Sociología (en inglés), 80, pp. 31-50.

Barnes J. A. (1954). «Class and Committee in a Norwegian Island Parish». Humans Relations, 7.

Bille H. T. (1995), «Measuring the Value of Culture». Revista Europea de Politica Cultural, vol. 1, n. . 2, pp. 309-322.

Bowman, C. y Ambrosini, V. (2000). «Value creation versus value capture: Towards a coherent definition of value in strategy». British Journal of Management, 11, pp. 1-15.

Buitrago, F, (2013). La economía naranja, una oportunidad infinita. Washington D. C.: Banco Interamericano de Desarrollo.

Castronova, E. (2006). Synthetic Worlds: The Business and Culture of Online Games. Chicago: University of Chicago Press.

Chan, S. (2001). Complex Adaptive Systems. ESD.83 Research Seminar in Engineering Systems. Disponible en: http:// web.mit.edu/esd.83/www/notebook/Complex\%20Adaptive $\% 20$ Systems.pdf.

Cunningham, S. (2007). A new economics for creative industries and development. WIPO, International conference on intellectual property and the creative industries.

Design Council (UK) (2004). «The Impact of Design on Stock Market Performance: An Analysis of UK Quoted Companies 1994-2003».

Design Taskforce (2003). «Success by Design NZ: A Report and Strategic Plan», Design Raskforce in Partnership with New Zealand Government, in support of the Growth and Innovation Framework (GIF). 
Devesa, M., BÁez, A., Figueroa, V. y Herrero, L. (2012). «Repercusiones económicas y sociales de los festivales cuturales: el caso del Festival Internacional de Cine de Valdivia». IEURE, vol. 38, n. ${ }^{\circ}$ 115. Disponible en: http://www.scielo. cl/pdf/eure/v38n115/art05.pdf.

Diment, K., Yu, P. y Garrety, K. (2012). «Complex Adaptive Systems as a Model for Evaluating Organisational: Change Caused by the Introduction of Health Information System». Health InformatICCs Research Lab, Faculty of InformatICCs, University of Wollongong, School of Management.

Goss, D. (2005). «Schumpeter's Legacy? Interaction and Emotions in the Sociology of Entrepreneurship». Entrepreneurship Theory and Practice, 29(2), pp. 205-219.

Hagoort, G. (2007). «Cultural Entrepreneurship. On the freedom to create art and the freedom of enterprise», conferencia inaugural, University of Utrecht.

HKU (2010). The Entrepreneurial Dimension of the Cultural and Creative Industries. Utrecht: Hogeschool voor de Kunsten Utrecht. Howard, D. y CROMPTON J. (2004). Financing Sport (2. ${ }^{a}$ ed.). West Virginia: Tecnología de Información sobre Fitness: Morgantown.

Howkins, J. (2001). The creative Economy. How People Make Money from Ideas. Londres: Penguin Books Limited.

Klamer, A. (1996). The Value of Culture, On the relationship between economICCs and arts. Ámsterdam: Amsterdam University Press. Kloudova, J. y Chwaszcz, O. (2014). «The analysis of the creative industry linked in connection with the economic development». E \& M Ekonomie a Management, 17(1), pp. 32-42.

Menger, C. (2007). El método de las ciencias sociales. Madrid: Unión Editorial.

Ministero de Cultura (2009). Manual de emprendimiento cultural. Bogotá: Ministerio de Cultura. 
Neugovsen, G. (2017). Creación de valor en las Industrias Culturales y Creativas. Buenos Aires: ESEADE.

Nicolas, Y. (2010). «El impacto económico de una actividad cultural como motivo de apoyo público: definición y condiciones de validez». Disponible en: https://www.cairn.info/ revue-d-economie-politique-2010-1-page-87.htm.

ORDÓÑEZ, D. (2006). La singularidad de los bienes creativos: aterrizando las diferencias, en Arte y parte: manual del emprendimiento en artes e industrias creativas. Bogotá: Ministerio de Cultura, Cámara de Comercio de Bogotá y British Council.

Phister, P. W. (2010). «Cyberspace: The Ultimate Complex Adaptive System». The International C2 Journal, vol. 4, n. ${ }^{\circ} 2$.

Potts, J. y Cunningham, S. (2007). «Four Models of the Creative Industries», CCI working paper, QUT.

Requena Santos, F. (2003). Análisis de redes sociales: orígenes, teorias y aplicaciones. Madrid: Alianza Editorial.

Schumpeter, J. A. (1934). The Theory of Economic Development. Cambridge, MA: Harvard University Press.

SeAman, B. (1987). «Estudios de impacto artístico: un exceso de moda». En RADICH, A. J. y Schwoch S. Impacto económico de las artes. Washington, D. C.: Conferencia Nacional de Legislaturas Estatales, pp. 43-75.

Stam, E., De Jong, J. y Marlet, G. (2008). Creative Industries in the Netherlands: Structure, Development, Innovativeness and Effects on Urban Growth. Geografiska Annaler: Series B.

TuluiN, P. (2012). «Why cultural entrepreneurship is a win-win scenario for the secton». Disponible en: https://www.theguardian.com/culture-professionals-network/culture-professionals-blog/2012/sep/24/cultural-entrepreneurship-technology-remix-ebook?goback=.gde_4726013_member_260900739.

Von Mises, L. (1949). Human Action: A Treatise on EconomICCs. New Haven: Yale University Press. 
De cadena de valor a sistemas adaptativos complejos en las industrias creativas y culturales

Wellman, B. (1988). «El análisis estructural de las redes sociales: del método y la metáfora a la teoría y la sustancia». Social Structures: A Network Approach. Cambridge University Press.

Neugovsen, Gerardo D. (2021). «De cadena de valor a sistemas adaptativos complejos en las industrias creativas y culturales». Monograma. Revista Iberoamericana de Cultura y Pensamiento, n. ${ }^{\circ}$ 9, pp. 81-117. doi: 10.36008/monograma.2021.09.2039. http: revistamonograma.com. ISSN: 2603-5839. 